\title{
A survey of codes and algorithms used in NERSC material science allocations
}

\author{
Lin-Wang Wang \\ NERSC System Architecture Team \\ Lawrence Berkeley National Laboratory
}

We have carried out a survey of codes and algorithms used on NERSC computers within the science category of material science. This is part of the effort to track the usage of different algorithms in NERSC community. This survey is based on the data provided in the ERCAP application of FY06. To figure out the usage of each code in one account, we have multiplied the total high performance computer (HPC) time allocation (MPP hours) of this account with the percentage usage of this code as estimated by the users in the ERCAP application. This is not the actual usage time, but should be a good estimation of it, and it represents the intention of the users. In a few cases $(\sim 5)$ where the estimated usages are not provided, rough values are used based on the numbers in similar projects and the author's experience in the related field. Different groups might have used slightly different names for a same code. This has been corrected manually by the author. The statistics is done based on the original allocation. The additional allocation after Bassi is online has roughly doubled the total allocation.

We have the following observations based on our survey:

(1) There are 65 accounts under the BES/material science category, they account for $20 \%$ of the 324 total NERSC accounts. The total HPC allocation for these accounts is 4.1 Mhours before the Bassi was online, 8.8Mhours after the Bassi was online. These account for $13 \%$ of the total NERSC allocations (66.7Mhours after the Bassi was online). This percentage is smaller than a few years ago, partly because the increase of some other categories, like fusion, and partly because the creations of special programs like SciDAC and INCITE.

(2) There are in total 62 major codes used in these 65 accounts, thus in average about one code per account (or say user group). However, since the same code can be used by different accounts, in average one code is used by 2.15 user groups. Except the VASP code, which is used by 23 groups, the majority of the codes is used by less than 5 groups, and many of the codes are used only by one group. This is a very diverse community, with many groups using their own codes.

(3) The different codes can be classified into 6 categories based on their physics and the corresponding algorithms. They are: DFT (density functional theory); beyond DFT (GW+BSE); QMC (quantum Monte Carlo); CMD (classical molecular dynamics); CMC (classical Monte Carlo); and other PDE (Partial differential equation). Their corresponding HPC time usages are: $74.0 \%, 6.9 \%, 6.7 \%, 6.4 \%$, $3.1 \%$, and $2.9 \%$, as represented in Fig. 1 . Thus, the majority of the time is spent on the DFT method, owing to the current success of that method in ab initio material science simulation. Within the DFT method, based on their different numerical 
approaches, they can be divided into: Plane Wave DFT, Green's function DFT, localized basis and orbital DFT, Maffin Tin sphere type DFT, and real space grid DFT (as listed in Table.II). The most popular (both in terms of number of codes and the HPC hours) one is the plane wave DFT. There are 12 codes for Planewave DFT, and account for 1.6 Mhours (before Bassi) (see Table.II). A more detailed explanation of these codes and algorithms will be given in the last paragraph.

All the 62 codes are listed in Table.I, along with the number of user groups, and the estimated HPC hours. A short description is also included for each code. This table is also presented as a plot in Fig.1. The data in Table.I has been regrouped in Table.II, divided into different types of codes, e.g, planewave LDA, localized orbital LDA, etc. Finally, the results in Table.II are summarized in Fig.2 and Fig.3.

As for mathematical algorithms and libraries, the information we can get from the ERCAP application is very limited. It might not be a reliable source to gauge which library is used and by what percentage of time. We do notice that many group indicate the usages of ESSL, fftw, lapack and scalapack. However, there is no information for which subroutines are used in these libraries, and by how much. But in general, we do feel that the above libraries are extremely important. At this point, we also do not know, for a typical material science code, how much time is spent on library routines, and how much time is spent on the rest of the code (e.g., the Fortran part written by users). Different extremes exist. For example, for a typical planewave DFT code, the majority of the time is spent on the user written Fortran code. But for a beyond DFT GW+BSE code, the majority of the time is spent on solving a dense linear algebra problem using, e.g, scalapack.

Finally, we like to provide a more detailed description of difference methods shown in Fig.3. This is most to help us to understand what are the relevant mathematical aspects and computer science issues. First, in the DFT (density functional theory) method, one needs to solve the single particle Schrodinger's equation (a second order partial differential equation). Typically $5-10 \%$ of the lowest eigen vectors are needed from this Schrodinger's equation (eigenstate equation). The number of eigen vectors is proportional to the number of electrons in the system. For example, for a thousand atom system, a few thousand eigen vectors are needed. This is a major difference to most engineering problems (e.g, fluid dynamics, climate simulation, combustion, where a small fixed number of time evolving fields are solved, and for Maxwell equation where a few electric magnetic eigen vector fields are solved). In DFT, the Schrodinger's equation (eigen state matrix) itself depends on the eigen vectors through the density function (thus the name of density functional theory). Thus it is a nonlinear problem. This nonlinear problem can be solved by selfconsistent iterations of the linearized eigenstate problem (Schrodinger's equation), or by direct nonlinear minimization. Currently, most large scale calculations are done using selfconsistent iterations. Numerically, what distinguish the different DFT methods and codes are the different basis sets used to describe the wavefunctions (the eigen vectors). Planewave DFT uses planewaves to describe the wavefunctions, while real space DFT uses a regular real space grid. Due to the sharp peak of the potential near the atomic nuclei, special cares are needed to choose different basis 
set. Besides the planewave and real space grid, the other conventional basis sets include: atomic orbital basis set where the eigen vectors of the atomic Schrodinger's equation are used to describe the wavefunctions in a solid or molecule; Gaussian basis set which is more often used in quantum chemistry due to its analytical properties; Muffin-tin basis where a spherical hole is cast out near each nuclei and spherical Harmonics and Bessel functions are used to describe the wavefunction inside the hole; Augmented planewaves where spherical Bessel functions near the nuclei are connected with the planewaves in the interstitial regions and used as the basis set; and the wavelet basis sets. In terms of the methods to solve the eigenstate problem, both iterative scheme and direct eigensolvers have been used in different codes. In the planewave DFT, iterative method (e.g, conjugated gradient method) is often used. While in the atomic orbital, Gaussian, and Augmented planewave (FLAPW) methods direct dense solvers (scalapack) are often used. For real-space grid method, sparse matrix solver is used. For the iterative solver, the most time consuming steps are the matrix vector multiplication and vector-vector multiplication (for orthogonalization). For the planewave DFT, the FFT is one bottleneck for large processor calculations.

$\mathrm{GW}+\mathrm{BSE}$ is one approach to calculate the excited states and optical spectrums. It requires large dense matrix. The most time consuming parts are to generate these matrix and diagonalize the matrix (for its eigen vectors). The diagonalization part is often done using dens eigen solver (scalapack). The dimension of the matrix is proportional to the square of the number of the electron in the system. Quantum Monte Carlo (MC) method uses stochastic random walk to carry out the multidimensional integral of the many body wavefunctions. Since it needs an assemble sum of different independent walkers, it is possible for embarrassing parallelization. Quantum MC is a very accurate method, but it suffers from statistical noises, thus it is difficult to be used for atomic dynamics (where the forces on the atoms are needed). For the classical molecular dynamics (MD), the parallelization is done in the step of force calculations. Since the classical force field formalism is local in nature (except the electrostatic force), efficient parallelization is possible as in the code of NAMD. Classical MC some time is used to replace the classical MD, thus it is more interested in the time evolving process, instead of an assemble sum (like in quantum MC). As a result, the parallelization is not so trivial. There are many recent developments for how to develop parallel schemes for classical MC (besides the possible approach for parallel evaluation of the total energy like in classical MD). Other PDE includes Maxwell equations (e.g, in photonic study), and time evolving differential equation for grain boundary and defect dynamics, etc. 


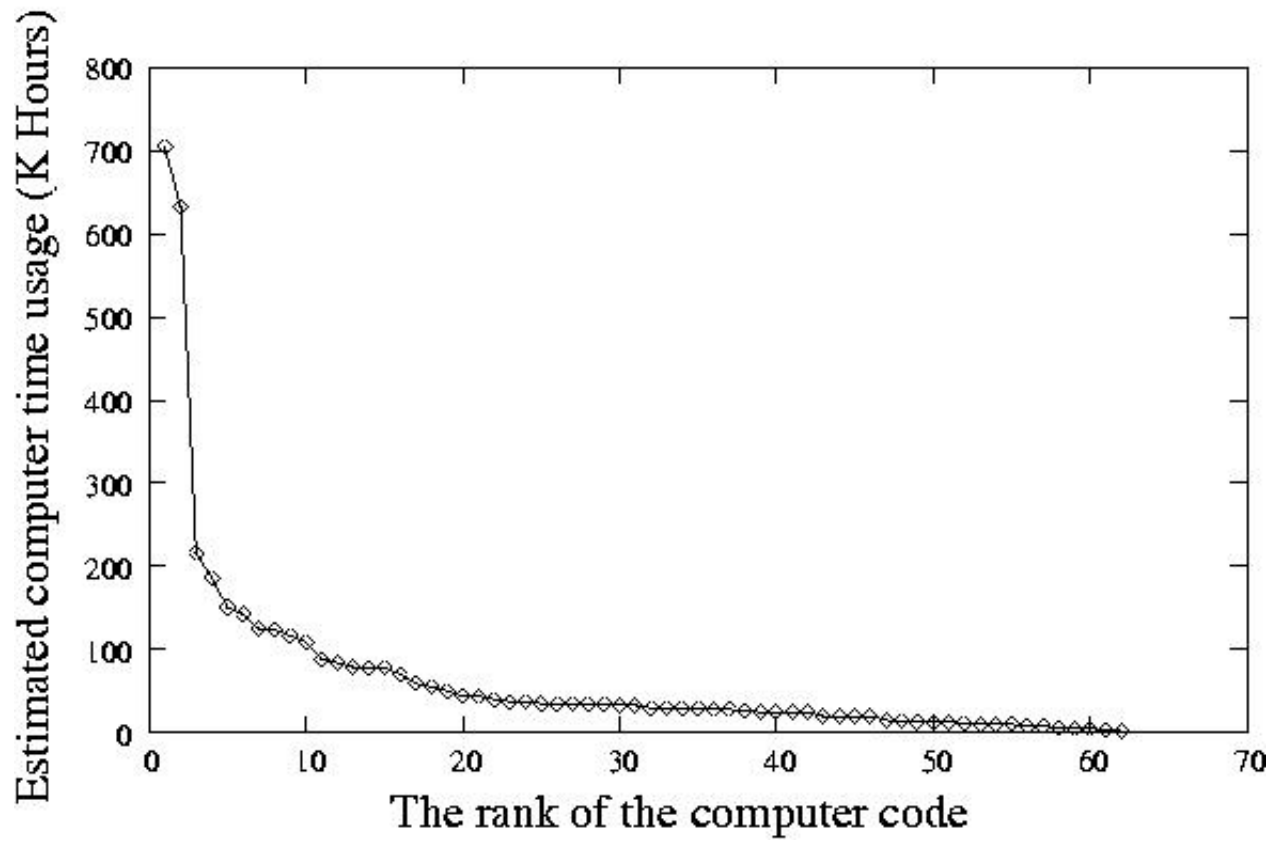

Fig.1, the computer time usage of different codes. Each symbol represents one code.

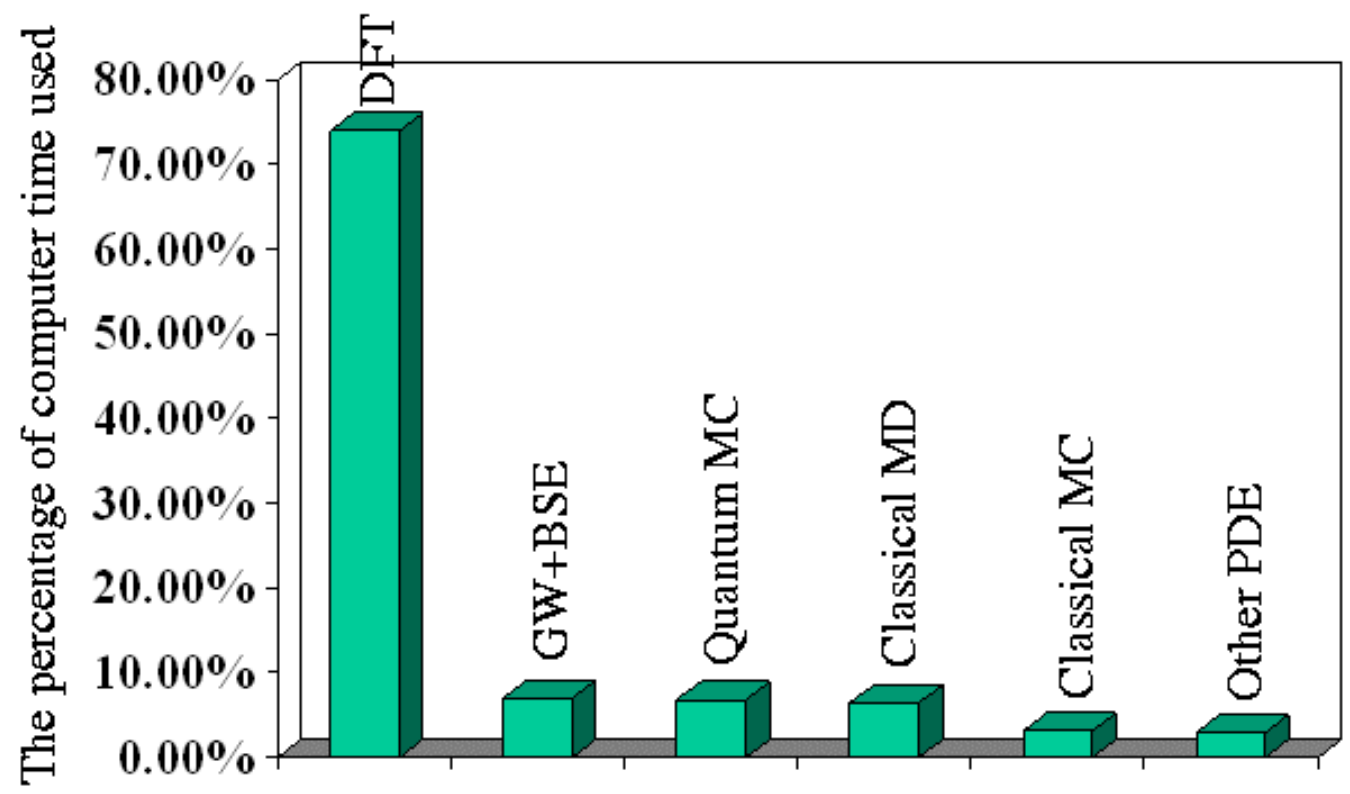

Fig.2: The percentage of computer time used for codes belong to different categories. The workload is dominated by Density Functional Theory (DFT) codes. 


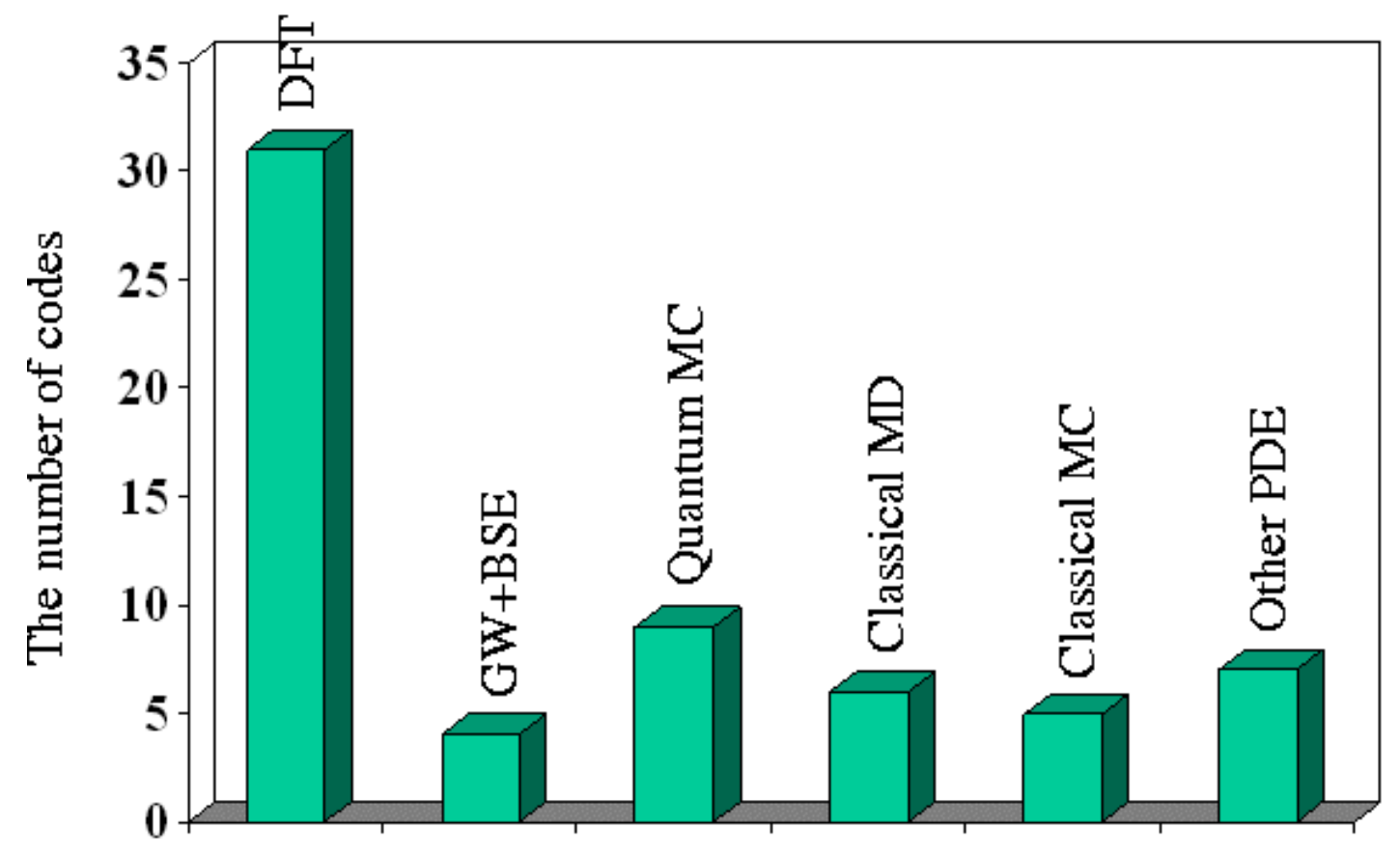

Fig.3: The number of codes belonging to different categories. 
Table.I, The list of all the codes used in Material Science on NERSC machines. $\mathrm{N}$-user is the number of groups (accounts) using that code. HPC $(\mathrm{KH})$ is the estimated high performance computer time (MPP hours) used for each code measured in thousand hours $(\mathrm{KH})$.

\begin{tabular}{|c|c|c|c|c|}
\hline rank & N-user & HPC(KH) & Code name & Narrative \\
\hline 1 & 23 & 704.5 & VASP & Vienna, planewave DFT \\
\hline 2 & 3 & 632.4 & LSMS & ORNL local mutiple scattering DFT \\
\hline 3 & 4 & 216.0 & PEscan & LBNL, planewave Folded Spectrum Method \\
\hline 4 & 3 & 186.0 & Scarlet & Berbeley, quantum transport, atomic basis \\
\hline 5 & 2 & 151.0 & ALCMD & Classical MD \\
\hline 6 & 6 & 142.5 & Paratec & Berloeley, planewave DFT \\
\hline 7 & 2 & 125.0 & Qbax & LLNL, massively parallel planewave DFT \\
\hline 8 & 3 & 124.0 & CMAT & NREL, Coulomb calc, and CI \\
\hline 9 & 6 & 116.5 & PWSCF & Planewave DFT \\
\hline 10 & 2 & 109.0 & FLAPW & NW Univ, FLAPW \\
\hline 11 & 4 & 89.00 & GW & Berbeley, GW \\
\hline 12 & 2 & 84.0 & PEtot & LBNL, planewave DFT \\
\hline 13 & 4 & 79.0 & SIESTA & Spain, local basis/orbital DFT \\
\hline 14 & 3 & 78.75 & TBMD & Tight-binding Molecular Dynamics \\
\hline 15 & 2 & 78.75 & BO-LSD-MD & Planewave DFT MD \\
\hline 16 & 1 & 70.0 & CASINO & Quantum MC \\
\hline 17 & 3 & 59.0 & DLPOLY & Classical MD \\
\hline 18 & 4 & 55.5 & NWchem & NWchem DFT, planeway and local basis \\
\hline 19 & 1 & 50.0 & CHAMP & Quantum Monte Carlo \\
\hline 20 & 2 & 43.75 & Multigrid & North Carolina, real space DFT \\
\hline 21 & 1 & 43.75 & XqmmmX & $\mathrm{QM} / \mathrm{MM}, \mathrm{PW}$ for QM, Amber for MM \\
\hline 22 & 1 & 40.0 & SSEqme & Quamtum MC for lattice spin \\
\hline 23 & 3 & 37.5 & Parsec & Minnesota, real space DFT \\
\hline 24 & 3 & 37.5 & RGWBS & Minnesota, G-space TDLDA \\
\hline 25 & 1 & 35.0 & LMTO-SIC & LMTO, DFT, Self-interaction correction \\
\hline 26 & 1 & 35.0 & Psi-Mag & Classical spin-MC \\
\hline 27 & 1 & 35.0 & SGF & Surface green's function \\
\hline 28 & 1 & 35.0 & Moldy & Force field classical MC \\
\hline 29 & 1 & 35.0 & OLCAO & LCAO TB like DFT \\
\hline 30 & 3 & 33.0 & NAMD & Classical force field MD \\
\hline 31 & 3 & 33.0 & BSE & Berbeley, Bethe-Salpeter Eq. \\
\hline 32 & 2 & 30.0 & FDTDn & Genetic Algorithm, classical el-mag, energy \\
\hline 33 & 1 & 30.0 & Flair & FLAPW LDA \\
\hline 34 & 1 & 30.0 & CF-Monte-Carlo & Quatum Monte Carlo for model $\mathrm{H}$ \\
\hline 35 & 2 & 30.0 & Abinit & Planewave DFT \\
\hline 36 & 1 & 28.0 & Dmol3 & Atomic orbital DFT \\
\hline 37 & 1 & 28.0 & FLMTO & LMTO DFT \\
\hline 38 & 1 & 26.0 & Polycrys & Plastic Deformation, grain boundary \\
\hline 39 & 1 & 25.0 & LAMMPS & Particle method molecular dynamics \\
\hline 40 & 1 & 25.0 & PWDFT & Planewave DFT \\
\hline 41 & 1 & 25.0 & QMC-DCA & Quantum MC and dynamic cluster \\
\hline 42 & 1 & 25.0 & SPF & Classical spin-phonon-Fermion MC \\
\hline 43 & 1 & 20.0 & Qdot & Quantum MC for 2D electron \\
\hline 44 & 1 & 20.0 & FEFF & Multi-scattering Green's func. electronic st \\
\hline 45 & 1 & 20.0 & becmw & Ordinary Differential Equation. \\
\hline 46 & 1 & 20.0 & Qmhubbard & Quantum Mechanics, hubbard model \\
\hline 47 & 1 & 15.0 & Freepar $\mathrm{x}$ & Special algorithm Quantum MC \\
\hline 48 & 1 & 14.0 & TransG98 & Green's func. transport, Gaussian basis \\
\hline 49 & 1 & 12.5 & AndyS & George Tech. Planewave DFT \\
\hline 50 & 1 & 12.5 & CL-GCMD & Classical MD, complex liquid \\
\hline 51 & 1 & 12.5 & Hollicita & $2 \mathrm{D}$ vertices $\mathrm{PDE}$ \\
\hline 52 & 1 & 12.0 & Mol-dyn & London Eq. MD, particle method \\
\hline 53 & 1 & 10.0 & Moment & Maxwell Eq, FFT, photonics \\
\hline 54 & 1 & 10.0 & TMM & Transfer matrix for Maxwell Eq, photonic \\
\hline 55 & 1 & 10.0 & BEST & Planewave DFT \\
\hline 56 & 2 & 8.0 & AMber & Classical force field MD \\
\hline 57 & 1 & 8.0 & $\mathrm{MC}$ & Classical MC for vortices \\
\hline 58 & 1 & 6.0 & ARPEsmpi & Multiple scat tering photoemission \\
\hline 59 & 1 & 5.0 & $\mathrm{AFQMC}$ & William Mary, Quantum Monte Carlo \\
\hline 60 & 1 & 5.0 & WIEN2K & Vienna, FLAPW \\
\hline 61 & 1 & 3.75 & Hartree & Real-space Hubbard Model, FH, LDA \\
\hline 62 & 1 & 2.0 & Ginger & Classical particle \\
\hline Total & & 4100.0 & & \\
\hline
\end{tabular}


Table.II, the computer codes in Table.I grouped into different categories.

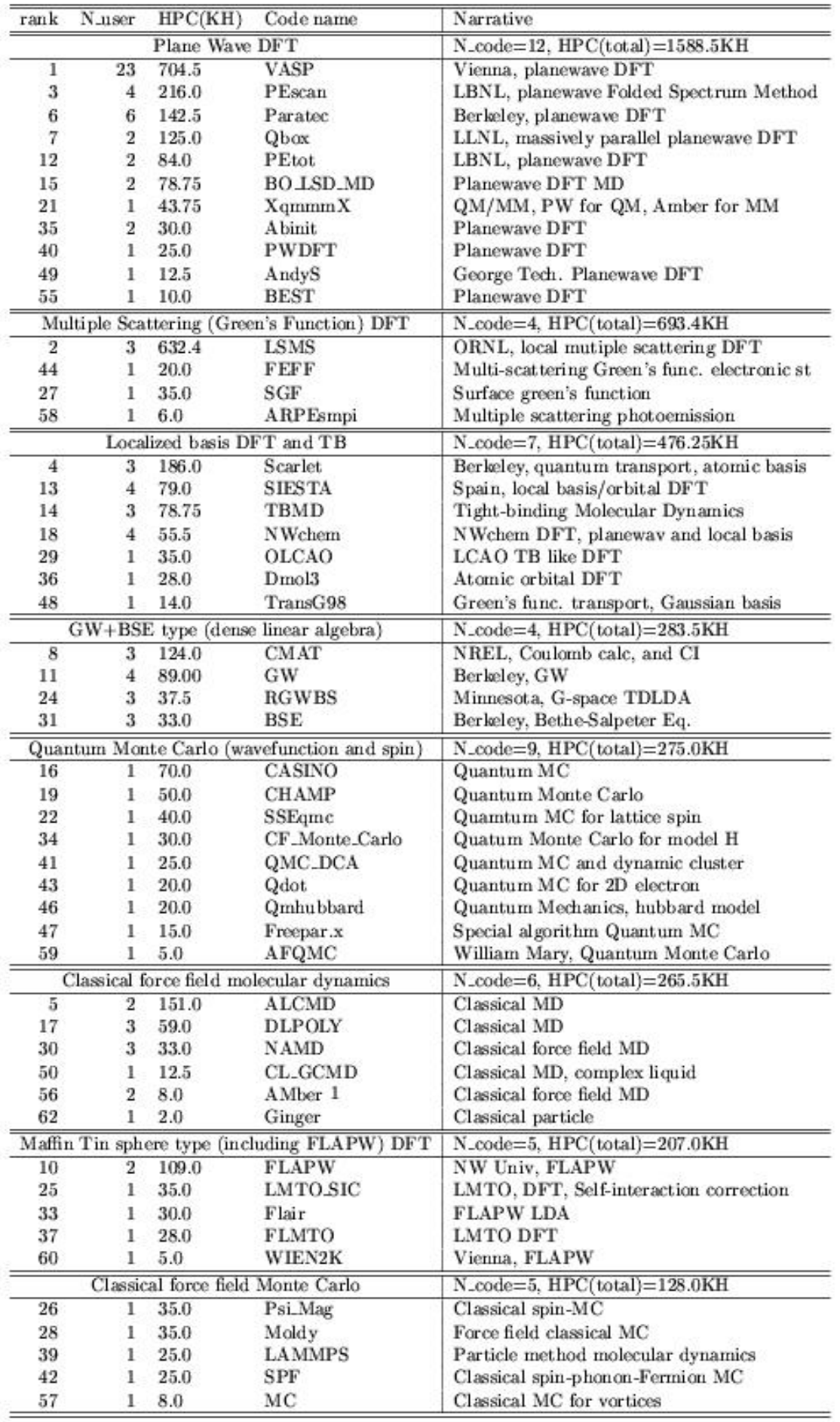




\begin{tabular}{cccl|l}
\hline \hline \multicolumn{9}{c}{ continue } \\
\hline rank & N_user & HPC(KH) & Code name & Narrative \\
\hline \hline \multicolumn{3}{c}{ Other PDE (many real space grid) } & N_code=7, HPC(total)=120.5KH \\
\hline 32 & 2 & 30.0 & FDTDn & Genetic Algorithm, classical el-mag, energy \\
38 & 1 & 26.0 & Polycrys & Plastic Deformation, grain boundary \\
45 & 1 & 20.0 & becmw & Ordinary Differential Equation. \\
51 & 1 & 12.5 & Hollicita & 2D vertices PDE \\
52 & 1 & 12.0 & Mol_dyn & London Eq. MD, particle method \\
53 & 1 & 10.0 & Moment & Maxuell Eq, FFT, photonics \\
54 & 1 & 10.0 & TMM & Transfer matrix for Maxuell Eq, photonic \\
\hline \hline \multicolumn{7}{c|}{ Real space grid DFT } & N_eode=3, HPC(total)=85.0KH \\
\hline 20 & 2 & 43.75 & Multigrid & North Carolina, real space DFT \\
23 & 3 & 37.5 & Parsec & Minnesota, real space DFT \\
61 & 1 & 3.75 & Hartroe & Real-space Hubbard Model, HF, LDA \\
\hline \hline Total & 4100.0 & \\
\hline \hline
\end{tabular}

